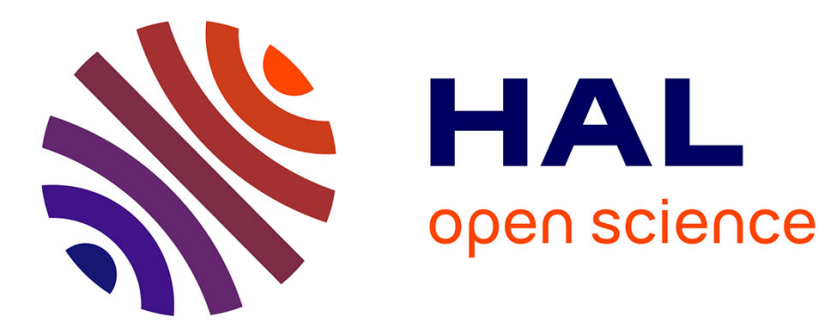

\title{
Cavitation in compressible visco-plastic materials
}

Christophe Denoual, Jean-Marc Diani

\section{To cite this version:}

Christophe Denoual, Jean-Marc Diani. Cavitation in compressible visco-plastic materials. Shock Compression of Condensed Matter-2001: 12th APS Topical Conference, Jun 2001, Atlanta, United States. pp.495-498. hal-00512434

\section{HAL Id: hal-00512434 https://hal.science/hal-00512434}

Submitted on 30 Aug 2010

HAL is a multi-disciplinary open access archive for the deposit and dissemination of scientific research documents, whether they are published or not. The documents may come from teaching and research institutions in France or abroad, or from public or private research centers.
L'archive ouverte pluridisciplinaire HAL, est destinée au dépôt et à la diffusion de documents scientifiques de niveau recherche, publiés ou non, émanant des établissements d'enseignement et de recherche français ou étrangers, des laboratoires publics ou privés. 


\title{
CAVITATION IN COMPRESSIBLE VISCO-PLASTIC MATERIALS
}

\author{
C. Denoual and J. M. Diani \\ Département de Physique Théorique et Appliquée. \\ CEA/DAM, BP 12, F-91680 Bruyères le Châtel Cedex, France.
}

\begin{abstract}
Ductile metals subjected to a high strain rate tensile loading are known to break by coalescence of voids. An analytical solution for the evolution of the cavity is proposed for compressible visco-plastic materials. Comparisons with FE calculations are proposed.
\end{abstract}

\section{INTRODUCTION}

The main mechanism leading to failure of ductile materials in hydrostatic tension is a cavitation instability (1), i.e., nucleation and coalescence of voids. The role of inertial effects $(2)(3)$ as well as the influence of triaxiality $(4)(5)$ or thermal softening $(6)$ is now well understood. Cavitation for compressible materials has also been studied for nonlinear elastic materials (7), but the influence of compressibility on elastic viscoplastic material still remains of interest. The aim of this study is to provide an evolution law for cavities in elastic visco-plastic materials that can be used in a finite element (FE) code and to understand the influence of compressibility on the overall behavior.

Cavitation instability is usually defined as the stress and strain state at which the growth of an infinitesimal cavity subjected to a tensile loading becomes unbounded (8), i.e., the loading is a prescribed pressure $P$ and no solution exists when $P \geq P_{\text {cr }}$ with $P_{\text {cr }}$ being the pressure to cavitation. However, when viscous behavior is considered, any pressure can be sustained by a sufficiently large strain rate. In this case the previous definition can no longer be used and cavitation criteria involving a threshold porosity for which macroscopic failure or pore coalescence are observed have to be proposed (9).

During a plate impact in spalling configuration, the stress in the damaged zone is mainly driven by inertial effects, i.e., the cavity radius does not tends toward infinity when $P>P_{\mathrm{cr}}$. The applied loading can rather be compared to a prescribed deformation. When such a macroscopic deformation is imposed, cavitation instability can be defined by

$$
P_{\mathrm{cr}}=P\left(t_{\mathrm{cr}}\right) \text { with } t_{\mathrm{cr}}: \frac{\partial P(t)}{\partial t}\left(t_{\mathrm{cr}}\right)=0
$$

where $t_{\mathrm{cr}}$ is the time to cavitation. Contrary to the definition where an unbounded growth of the cavity is expected (in the case of an applied pressure), an ultimate macroscopic pressure is obtained by using Eqn. (1) even for viscous materials. This critical pressure $P_{\text {cr }}$ can be compared to the ultimate stress measured during a plate impact experiment in spalling configuration. Instability of a micro void in a matrix with an elastic visco-plastic behavior is studied in the following sections. After a presentation of the constitutive equations, the role of viscosity and compressibility of the material is analyzed.

Numerical estimations are performed by using a copper-like material (bulk modulus $=131 \mathrm{GPa}$, shear modulus $=47 \mathrm{GPa}$, Yield stress $=500 \mathrm{MPa}$, mass density $=8960 \mathrm{Kg} / \mathrm{m}^{3}$ ). Since this study aims at showing the effect of viscosity on the cavitation mechanism, the viscosity parameter $\eta$ is tuned in order to explore different regimes of cavitation.

\section{CONSTITUTIVE EQUATIONS}

Due to the high pressure reached before instability, the bulk expansion may represent an important amount of energy stored around the cavity. This energy is suspected to be released when the cavity nucleates and 
metallic materials. Unfortunately, the equations that describe the strain and stress around the cavity are difficult to handle when pressure is considered. Since an exact solution cannot be exhibited, the following approximation for the displacement field is proposed

$$
r^{3}=\varphi(t)\left[r_{0}^{3}+\omega(t)\right]
$$

where the parameter $\omega(t)$ stands for the change in volume of the cavity and $r\left(r_{0}, t\right)$ is the Lagrangian radius at time $t$ and $r_{0}$ the radius at $t=0$. One observes that the proposed displacement field is an incompressible one modified by a dilatation of factor $\varphi(t)$. The velocity $\boldsymbol{v}$ is defined by

$$
\boldsymbol{v}\left(r_{0}, t\right)=\frac{\mathrm{d} r\left(r_{0}, t\right)}{\mathrm{d} t} \boldsymbol{e}_{\mathrm{r}}=\left(\frac{\varphi \dot{\omega}}{3 r^{2}}+\frac{r}{3} \frac{\dot{\varphi}}{\varphi}\right) \boldsymbol{e}_{\mathrm{r}}
$$

The mean and deviatoric part of the strain rate $\mathrm{d}=$ $\frac{1}{2}\left(\nabla \boldsymbol{v}+\nabla^{\mathrm{t}} \boldsymbol{v}\right)$ can be easily deduced from Eqn (3)

$$
\begin{aligned}
& d_{\mathrm{eq}}=\frac{2}{3}\left|d_{\theta \theta}-d_{\mathrm{rr}}\right|=\frac{2}{3} \frac{\varphi \dot{\omega}}{r^{3}} \\
& d_{\mathrm{m}}=\frac{1}{3}\left(d_{\mathrm{rr}}+2 d_{\theta \theta}\right)=\frac{1}{3} \frac{\dot{\varphi}}{\varphi}
\end{aligned}
$$

where the subscripts "rr" refer to radial components and " $\theta \theta$ " to the circumferential ones. The elastic strain tensor $\epsilon$ refers to small strain and can approximated as follow

$$
\epsilon=\epsilon^{\mathrm{e}}+\epsilon^{\mathrm{p}} \approx \frac{1}{2}\left(\nabla \boldsymbol{u}+\nabla^{\mathrm{t}} \boldsymbol{u}\right)=\int_{0}^{\mathrm{t}} \mathrm{d}^{\mathrm{e}}+\mathrm{d}^{\mathrm{p}} \mathrm{dt}
$$

where the superscripts " $e$ " and "p" denote the elastic and plastic parts respectively, and where $\boldsymbol{u}$ is the displacement vector $\left(u_{\mathrm{r}}=r(t)-r(0)\right)$.

The stress-strain relationship is defined by linear elasticity

$$
\sigma_{i j}^{\mathrm{e}}(r, t)=2 G \varepsilon_{i j}^{\mathrm{e}}(r, t)+\left(K-\frac{2}{3} G\right) \varepsilon_{k k}^{\mathrm{e}}(r, t) \delta_{i j}
$$

where $G$ is the shear modulus and $K$ the bulk modulus. In the elastic part $\left(r>r_{\mathrm{p}}\right)$, the mean $\left(\sigma_{\mathrm{m}}^{\mathrm{e}}=\right.$ $\left.\frac{1}{3}\left(\sigma_{\mathrm{rr}}+2 \sigma_{\theta \theta}\right)\right)$ and deviatoric $\left(\sigma_{\mathrm{eq}}^{\mathrm{e}}=\left|\sigma_{\theta \theta}-\sigma_{\mathrm{rr}}\right|\right)$ stresses are obtained by using Eqns (6), (7)

$$
\begin{aligned}
\sigma_{\mathrm{eq}}^{\mathrm{e}} & =2 G \ln \left(\frac{r^{3}}{r^{3}-\varphi \omega}\right) \approx 2 G\left(\frac{\varphi \omega}{r^{3}-\varphi \omega}\right) \\
\sigma_{\mathrm{m}}^{\mathrm{e}} & =K \ln (\varphi)
\end{aligned}
$$

For metallic materials below the elastic limit, the stress field proposed in Eqns (8) and (9) is the exact solution under the assumption of small deviatoric displacement field $(\varphi \omega \ll 1)$. The radius $r_{\mathrm{p} 0}^{3}=\varphi^{-1} r_{\mathrm{p}}^{3}-\omega$ associated to the transition from elasticity to plasticity is defined by $\sigma_{\text {eq }}\left(r_{\mathrm{p}}, t\right)=Y_{0}$

$$
r_{\mathrm{p} 0}^{3}=\frac{2 G \omega}{Y_{0}} \text { if } \omega \geq a_{0}^{3} \frac{Y_{0}}{2 G} \text { and } \omega<b_{0}^{3} \frac{Y_{0}}{2 G}
$$

$r_{\mathrm{p} 0}^{3}=a_{0}^{3}$ (resp. $b_{0}^{3}$ ) when $\omega<a_{0}^{3} Y_{0} / 2 G$ (resp. $\omega>$ $\left.b_{0}^{3} Y_{0} / 2 G\right)$.

In order to obtain analytical results, a Bingham law for elastic visco-plastic material is chosen (only loading conditions are considered hereafter)

$$
\sigma_{\mathrm{eq}}(r, t)=Y_{0}+\eta d_{\mathrm{eq}}^{\mathrm{p}}(r, t) \quad \text { when } \quad r<r_{\mathrm{p}}
$$

where $Y_{0}$ is the yield stress, $d_{\mathrm{eq}}^{\mathrm{p}}$ the equivalent plastic strain rate and $\eta$ is the viscosity parameter.

\section{APPROXIMATED MACROSCOPIC PRESSURE}

Inertial effects are assumed to have a weak influence on $P_{\mathrm{cr}}$. The equation of equilibrium in spherical geometry therefore is

$$
\frac{\partial \sigma_{\mathrm{rr}}}{\partial r}+\frac{2}{r}\left(\sigma_{\mathrm{rr}}-\sigma_{\theta \theta}\right)=0
$$

This leads to an expression for $\sigma_{\mathrm{rr}}(r)$

$$
\begin{aligned}
\frac{3}{2} \frac{\sigma_{\mathrm{rr}}(r)}{Y_{0}}= & \varphi \omega \frac{2 G}{Y_{0}}\left(\frac{1}{r_{\mathrm{p}}^{3}}-\frac{1}{r_{1}^{3}}\right) \\
& +\int_{a^{3}}^{r_{2}^{3}}\left[1+\frac{\eta}{Y_{0}} d_{\mathrm{eq}}^{\mathrm{p}}(r, t)\right] \frac{\mathrm{dr}^{3}}{r^{3}}
\end{aligned}
$$

with $r_{1}=\max \left(r, r_{\mathrm{p}}\right)$ and $r_{2}=\min \left(r, r_{\mathrm{p}}\right)$. The macroscopic pressure $P=\sigma_{\mathrm{rr}}(b)$ is obtained by using Eqn. (13) with the assumption that the plastic and total deviatoric strain rates are comparable $\left(d_{\mathrm{eq}}^{\mathrm{p}} \approx d_{\mathrm{eq}}=\right.$ $\left.\frac{2}{3} \frac{\varphi \dot{\omega}}{r^{3}}\right)$

$$
\begin{aligned}
\frac{3}{2} \frac{P}{Y_{0}}=\varphi \omega \frac{2 G}{Y_{0}}\left(\frac{1}{r_{\mathrm{p}}^{3}}-\frac{1}{b^{3}}\right) & +\ln \left(\frac{r_{\mathrm{p}}^{3}}{a^{3}}\right) \\
& -\frac{2}{3} \frac{\eta}{Y_{0}} \varphi \dot{\omega}\left(\frac{1}{r_{\mathrm{p}}^{3}}-\frac{1}{a^{3}}\right)
\end{aligned}
$$

with $a \leq r_{\mathrm{p}} \leq b$. The only unknowns in the Eqn. (14) are $\dot{\omega}$ and $\dot{\varphi}$. 


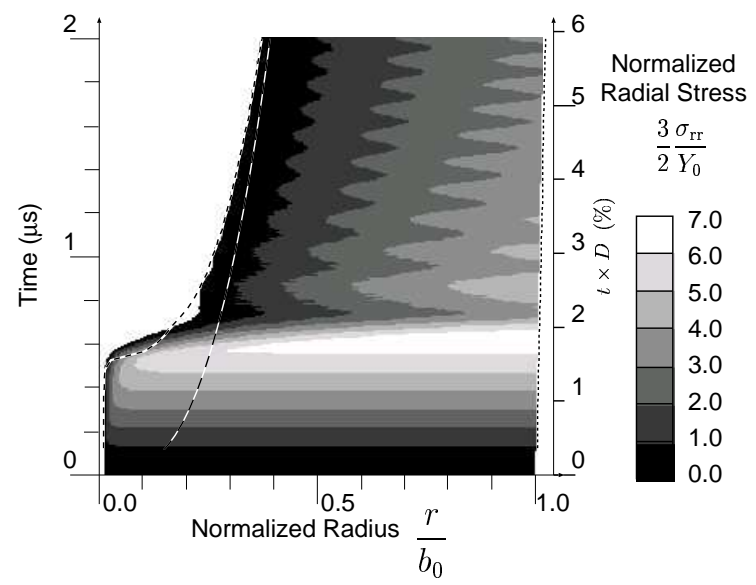

FIGURE 1. Space-time diagram of cavitation mechanism $\left(f_{0}=\right.$ $10^{-6}, D=3 \times 10^{4} \mathrm{~s}^{-1}$ ) given by the proposed differential equation (dotted lines stand for inner and outer radii) and by the FE code (grey colored zone). The left white zone represents the cavity and the right one is the outer boundary. The left and right dotted lines are obtained by using Eqns. (18) and (2), the middle dashed line is the radius $a(t)$ estimated by using a hypothesis of incompressibility $(\varphi=1)$.

A first relationship between $\dot{\omega}$ and $\dot{\varphi}$ is given by the displacement boundary condition corresponding to a prescribed hydrostatic strain rate $D$

$$
D=3 \frac{v_{\mathrm{r}=\mathrm{b}}}{b}=\frac{\varphi \dot{\omega}}{b^{3}}+\frac{\dot{\varphi}}{\varphi}
$$

The second relationship is given by using the definition of radial stress $\sigma_{\mathrm{rr}}$ at $r=r_{\mathrm{p}}$ with both Eqns. (7) (with $\sigma_{\mathrm{eq}}^{\mathrm{e}} / Y_{0}=1$ ) and (13)

$$
\begin{aligned}
\frac{3}{2} \frac{\sigma_{\mathrm{rr}}\left(r_{\mathrm{p}}\right)}{Y_{0}} & =\int_{a^{3}}^{r_{\mathrm{p}}^{3}}\left(1+\frac{2}{3} \frac{\eta}{Y_{0}} \frac{\varphi \dot{\omega}}{r^{3}}\right) \frac{\mathrm{dr}^{3}}{r^{3}} \\
& =\frac{3}{2} \frac{K}{Y_{0}} \ln (\varphi)-1
\end{aligned}
$$

with $a \leq r_{\mathrm{p}} \leq b$. The evolution of $\dot{\omega}$ can be performed when $r_{\mathrm{p}} \geq a(t)$, i.e., when

$$
\omega \geq a_{0}^{3} \frac{Y_{0}}{2 G} \quad \text { and } \quad \varphi \geq \frac{2}{3} \frac{Y_{0}}{K}+1
$$

(see Eqn. (10) and Eqn. (16) with $a=r_{\mathrm{p}}$ ).

\section{APPLICATIONS}

The evolution of the pressure $P=\sigma_{\mathrm{rr}}(b)$ is computed in the following sections by using a firstorder differential equation on $\omega$ obtained by using Eqns. (15), (16) and (17) for elastic-plastic material and elastic-plastic materials with viscosity. The FE calculations are performed for compressible materials by using a Lagrangian 1D code with Alternate Lagrangian Eulerian scheme (ALE). For all the calculations, the node density is chosen to be equal to $20 / a_{0}$ at $t=0$ and the hydrostatic strain rate is $3 \times 10^{4} \mathrm{~s}^{-1}$. The dimension of the initial radius $b_{0}$ is chosen so that inertial effects have a weak influence on the pressure to cavitation $P_{\text {cr }}\left(b_{0} \approx 0.1 \mathrm{~mm}\right)$.

\section{Elastic plastic material}

Equation (16) used with $\eta=0$ together with Eqn. (15) lead to the following differential equation

$$
\dot{\bar{\omega}}\left(t>t_{0}\right)=D \frac{\left(f_{0}+\bar{\omega}\right)(1+\bar{\omega})}{\left(f_{0}+\bar{\omega}\right)+\xi}
$$

with $\bar{\omega}=\omega / b_{0}^{3}$ and $\omega\left(t_{0}\right)=\omega_{0}$ and

$$
\xi=\left\{\begin{array}{lll}
\frac{2}{3} \frac{Y_{0}}{K} f_{0}\left(1+\frac{1}{\bar{\omega}}\right) & \text { if } \quad \bar{\omega}<\frac{Y_{0}}{2 G} \\
\frac{2}{3} \frac{Y_{0}}{K}\left(f_{0}-1\right) & \text { if } \quad \bar{\omega} \geq \frac{Y_{0}}{2 G}
\end{array}\right.
$$

The evolution of $\varphi$ is given by Eqn. (15).

An example of evolution of the inner and outer radii is given in Fig. 1 and can be described in three stages: nucleation, inflation and growth. During the nucleation stage (a few tenth of microseconds), the stress $\sigma_{\mathrm{rr}}$ is homogeneous over the volume but in a small zone surrounding the cavity, hardly visible when the whole sphere is considered. When $t \approx 0.5 \mu \mathrm{s}$ $(t \times D \approx 1.5)$ a dramatic increase of the cavity radius can be observed, followed by a pressure drop. The calculated strain field then is no longer comparable to the quasi-static one and bouncing of the material surrounding the cavity can be observed at $0.4 \mu \mathrm{s}$. This stage where the stress/strain state jumps as fast has possible from the quasi-static solution to the incompressible one (the middle dashed line in Fig. 1) can be termed the inflation stage. Finally, during the growth stage the evolution of the internal radius is comparable to that given by an incompressible displacement field $(\varphi=1)$.

\section{Elastic visco-plastic material}

Equation (16) with $\eta \neq 0$ then leads to the following differential equation in $\bar{\omega}$

$$
\dot{\bar{\omega}}=-\left(f_{0}+\bar{\omega}\right) \frac{3}{2} \frac{Y_{0}}{\eta}\left[1+\xi-\frac{3}{2} \frac{K}{Y_{0}} \ln (\varphi)\right]
$$




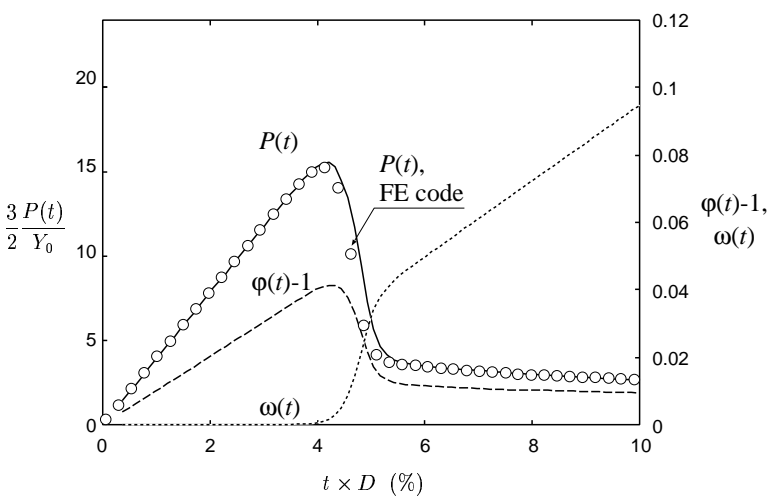

FIGURE 2. Evolution of pressure vs. hydrostatic deformation for a compressible elastic visco-plastic material $\left(f_{0}=10^{-6}, \eta / Y_{0}=\right.$ $3 / 210^{-6}$ ) and the corresponding set of parameters $\omega$ and $\varphi$. Lines represent numerical estimations (Eqn (18)) and dots stand for FE calculation

with

$$
\xi= \begin{cases}\ln \left[\left(\frac{2 G}{Y_{0}}+1\right)\left(1-\frac{f_{0}}{f_{0}+\bar{\omega}}\right)\right] & \text { if } \bar{\omega}<\frac{Y_{0}}{2 G} \\ \ln \left(\frac{1+\bar{\omega}}{f_{0}+\bar{\omega}}\right) & \text { if } \bar{\omega} \geq \frac{Y_{0}}{2 G}\end{cases}
$$

Figure 2 shows the evolution of the pressure and parameters $\omega(t), \varphi(t)$ versus time. The pressure given by FE calculation shows the same features as depicted in Fig. 1 (that is, nucleation, inflation and growth) but nevertheless without oscillations in the so-called growth stage. During the nucleation stage, the cavity does not influence much the evolution of the pressure $P(t)$, which can be approximated by $K t D$. Since $\omega$ does not increase, the energy is stored to a great extent through hydrostatic strain. This energy is used during the inflation stage to make the cavity quickly grows ( $\varphi$ is decreasing when $\omega$ is increasing), as depicted in Fig. 2. Compressibility therefore acts as an energy buffer which fills up when the growth of the cavity is difficult, and emptied when the cavity growth becomes easy. Finally, during the growth stage, the cavity experiences a quasi-incompressible growth, with $\varphi$ remaining constant while $\omega$ linearly increases.

\section{CONCLUSIONS}

For an elastic-plastic material $(\eta=0)$, the critical pressure (plotted on Fig. 3) reaches a plateau when $f_{0}$ is decreasing, i.e., $P_{\mathrm{cr}}$ does not depend on the initial porosity if $f_{0}<10^{-5}$ and nucleation of voids can occur even for dense materials $\left(f_{0} \rightarrow 0\right)$. One can observe that the same numerical result can be obtained

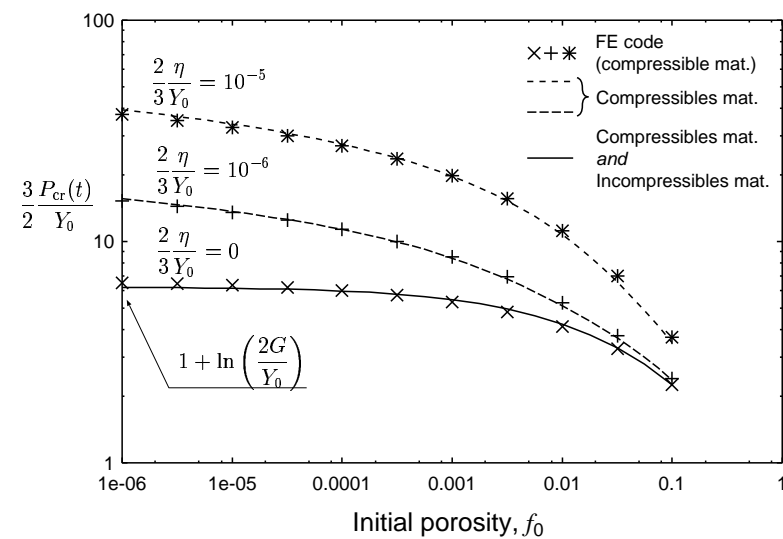

FIGURE 3. Normalized pressure to cavitation vs. initial porosity $f_{0}$. When no viscosity is used $(\eta=0)$ compressible and incompressible displacement fields for both FE calculations and analytical approach give the same numerical result.

with incompressible materials, i.e., with $\varphi=1$ as depicted in Fig. 3. In this case, pressure to cavitation for dense, compressible and perfectly plastic materials is found to be $\frac{3}{2} \frac{P_{\mathrm{cr}}}{Y_{0}}=1+\ln \frac{2 G}{Y_{0}}$ (by using Eqns. $(14,15)$ and (1) with $f_{0} \rightarrow 0$ ).

As expected, the ultimate pressure for viscous materials is far greater than that of elastic-plastic ones. For these materials, incompressibility cannot be used to describe the cavitation mechanism since $\varphi=1$ leads to $P \propto \eta / f$ when $f \rightarrow 0$, see Eqn. (14-15). Therefore, compressibility does not play any role for cavitation in elastic plastic materials but is the crux of the cavity evolution in elastic-visco-plastic ones.

For all the simulations, the analytical approach reproduces very well the finite element simulations. The proposed compressible displacement field and evolution laws might therefore be used as components of a more general damage model (10) in the spirit of Gurson's (11).

\section{REFERENCES}

1. J. M. Ball, Phil. Trans. R. Soc. Lond. A 306, 557 (1982).

2. M. M. Carroll and A. C. Holt, J. Appl. Phys. 43, 1626 (1972).

3. W. Tong and G. Ravichandran, J. Appl. Mech. 62, 633 (1995).

4. H. S. Hou and R. Abeyaratne, J. Mech. Phys. Solids 40, 571 (1992).

5. R. Cortes, J. Solids Structures 29, 1637 (1992).

6. R. Cortes, J. Solids Structures 29, 1339 (1992).

7. C. O. Horgan and R. Abeyaratne, J. Elasticity 16, 189 (1986).

8. Y. H. V. Tvergaard and J. W. Hutchinson, Eur. J. Mech., A/Solids 11, 215 (1992)

9. Z. P. Wang, J. Appl. Phys. 76, 1535 (1993).

10. C. Denoual, in preparation. .

11. A. L. Gurson, J. Eng. Mater. Technol. 2, 2 (1977). 